Meta

Journal des tradlucteurs

Translators' Journal

\title{
Émission, transmission et compagnie
}

\section{Robert Dubuc}

Volume 13, numéro 1, mars 1968

URI : https://id.erudit.org/iderudit/002607ar

DOI : https://doi.org/10.7202/002607ar

Aller au sommaire du numéro

Éditeur(s)

Les Presses de l'Université de Montréal

ISSN

0026-0452 (imprimé)

1492-1421 (numérique)

Découvrir la revue

Citer cet article

Dubuc, R. (1968). Émission, transmission et compagnie. Meta, 13(1), 22-24.

https://doi.org/10.7202/002607ar d'utilisation que vous pouvez consulter en ligne.

https://apropos.erudit.org/fr/usagers/politique-dutilisation/ 


\section{ÉMISSION, TRANSMISSION ET COMPAGNIE}

Les communications, dans les pays où il y a d'énormes distances, ont toujours pris une importance vitale. Les messages de fumée des Tsonnontouans et des Onneyouts ont annoncé d'une certaine façon les communications du Canada moderne, dont le phare hertzien (microwave tower) est devenu le symbole.

Ce phare symbolique ne représente qu'une étape dans la chaîne qui conduit du studio au poste récepteur les images et les sons qui peuplent notre civilisation du loisir. Partant du studio, où le réalisateur leur a donné forme, sons et images s'acheminent vers l'antenne émettrice (transmitting antenna) pour commencer leur pérégrination dans l'éther. L'antenne émettrice fait partie de l'émetteur (transmitter) qui comprend «l'ensemble des dispositifs et appareils destinés à produire des oscillations électriques, dont l'énergie est rayonnée à distance sous forme d'ondes électromagnétiques ${ }^{1}$.

On confond souvent en français, à l'instar des journalistes trop pressés, émetteur et transmetteur. Transmetteur désigne en français «l'appareil qui sert à transmettre les signaux télégraphiques $\gg^{2}$. L'anglais désigne ces deux réalités d'un même terme, transmitter.

La langue a bien des caprices qu'il n'est pas toujours facile d'expliquer. Comment se fait-il, par exemple, que la distinction qui s'est établie entre émetteur et transmetteur ne se soit pas transposée dans les noms d'action - émission et transmission - qui y correspondent ?

Émission désigne la diffusion, par l'émetteur, des images et des sons - selon le cas - traduits en ondes électromagnétiques et rayonnés dans l'espace. Transmission marque de son côté le voyage complet de l'onde, de l'émetteur au récepteur ${ }^{3}$. En ce sens, on dit correctement: ligne de transmission (transmission line), moyens de transmission (transmission media). Émission (broadcast) et transmission (transmission) représentent donc deux aspects distincts de la communication radiophonique ou télévisuelle.

Comme le changement est une manifestation de la vie, une langue vivante évolue et, avec elle, les mots qui la composent. Émission et transmission n'ont pas gardé exclusivement leurs sens primitifs tout près de leur étymologie. Leur sens s'est étendu à d'autres réalités voisines.

Nous avons parlé dans une chronique antérieure ${ }^{4}$ des sens dérivés d'émission. Nous allons nous arrêter maintenant aux extensions sémantiques que transmission a connues de son côté.

1. Jean Brun, Dictionnaire de la radio, Paris, Albin Michel, 1949, p. 208.

2. Transmetteur s'employait autrefois au sens d'émetteur. Mais aujourd'hui l'usage a spécialisé les deux termes en limitant transmetteur à la télégraphie et émetteur à la radiotélévision. Cf. à ce sujet l'article transmetteur, Larousse du $X X^{e}$ siècle, Paris, Larousse, 1953.

3. Jean Brun, op. cit., p. 503: «Transmission: Transfert d'un poste émetteur à un poste récepteur des signaux de nature quelconque comportant communication destinée à un ou plusieurs correspondants. "

4. "Programme ou émission", Journal des Traducteurs, vol. VIII, no 3, juillet-août 1963, p. 97. 
De l'action de transmettre, transmission a désigné la chose transmise au moyen d'un enregistrement. Cette acception ne laisse pas de surprendre un peu quand on en ignore l'origine. C'est d'abord la locution transmission différée (delayed broadcast) qui s'est employée en ce sens ${ }^{5}$. Puis, l'usage a laissé peu à peu tomber l'adjectif pour ne retenir que le mot transmission, comme en fait foi la définition donnée par $H$. Bénac ${ }^{6}$. Voici quelques exemples de cet emploi: "Que l'on se souvienne des correctifs qu'on a été obligé d'apporter à certaines transmissions d'ensemble avec solistes. » 7 ; $\ll \mathrm{La}$ transmission de plusieurs soirées $\mathrm{du}$ festival d'Aix-en-Provence est sans doute un petit événement de sociologie culturelle. $\otimes^{8}$

Quant à l'adjectif différé, il s'est substantivé au masculin pour désigner la diffusion d'un enregistrement d'émission (delayed broadcasting). "Pourquoi fait-on ces réalisations en direct alors qu'on pourrait les faire en différé ? ${ }^{9}$ Le direct (live broadcasting) s'oppose donc au différé pour désigner deux procédés de transmission au sens strict.

Poursuivant notre recensement, nous tombons sur un dérivé itératif de transmission: retransmission. Comme on peut s'y attendre, ce mot signifie d'abord transmettre de nouveau. Ce dérivé s'est toutefois enrichi à son tour d'un sens nouveau, assez difficilement explicable, mais apparemment très courant: «Diffusion d'une manifestation publique, en dehors du studio, qui n'était pas spécialement organisée pour la T.S.F. ${ }^{10}$ Le verbe retransmettre a aussi la même acception. Citons quelques exemples: «Faut-il supprimer les retransmissions théâtrales?» ${ }^{11}$; «La retransmission de manifestations sportives ... ${ }^{12}$; $\ll \mathrm{Le}$ concert est retransmis de la salle X.» ${ }^{13}$; «Music-Hall Parade [est] retransmis du Moulin de la Galette. ${ }^{14}$

Pour résumer, disons que transmission, dans son sens dérivé, s'applique au différé, et retransmission, au direct. L'anglais ne fait pas cette distinction. Dans les deux cas, on emploie remote broadcast, quitte à préciser au besoin par les qualificatifs delayed ou live.

Au Canada français, retransmission, en ce dernier sens, est à peu près inusité. On emploie plutôt radiodiffusion et télédiffusion: «La télédiffusion d'un match de hockey. » Cet usage comporte des inconvénients. D'abord, dans le cas de radiodiffusion, il y a perte d'information; retransmission comporte une précision que radiodiffusion n'implique pas, à savoir que «l'événement n'est pas spéciale-

5. L'expression transmission différée se dit d'une émission radiophonique (ou télévisuelle) qui a été enregistrée pour être diffusée ultérieurement. Cf. Larousse universel, p. 954.

6. Dictionnaire des synonymes, Paris, Hachette, 1956, p. 305: "Diffusion, après coup, d'un ensemble sonore enregistré. »

7. Cahiers d'étude de la radio-télévision, $\mathrm{n}^{\circ} 18, \mathrm{p} .167$.

8. Jean Quéval, Nouvelles littéraires, 30 juillet 1959.

9. Paul Vialar, Nouvelles littéraires, 18 octobre 1962.

10. H. Bénac, op. cit., p. 305.

11. Combat, 9 septembre 1959.

12. Cahiers d'étude de la radio-télévision, $\mathrm{n}^{\circ} 2, \mathbf{p} .193$.

13. Adolphe $\mathrm{V}$. Thomas, Dictionnaire des difficultés de la langue française, Paris, Larousse, 1956, p. 124

14. Télémagazine, $\mathrm{n}^{\circ} 102$. 
ment organisé pour la radio ». Quant à l'emploi de télédiffusion, dont nous avons déjà parlé ${ }^{15}$, il constitue une impropriété de terme qui va à l'encontre de l'usage international. Ici encore, nous aurions profit à rejeter ces particularismes inutiles qui nous coupent de la communauté francophone. Nous n'y trouvons qu'affaiblissement.

Robert Dubuc 